\title{
Antibiotikaprophylaxe kann den Outcome nicht verbessern
}

Fragestellung: Ist eine präventive Behandlung mit Antibiotika bei Patienten mit akutem Schlaganfall wirksam?

Hintergrund: Bei etwa $30 \%$ aller $\mathrm{Pa}$ tienten, die einen akuten ischämischen Insult oder eine zerebrale Blutung erleiden, kommt es konsekutiv zu einer Infektion. Meist handelt es sich um bakterielle Pneumonien oder Harnwegsinfekte. Fieberhafte Infekte nach einem Schlaganfall sind ein ungünstiger prognostischer Faktor. Bisher gibt es fünf kleinere Studien, die untersucht haben, ob eine präventive Antibiose den funktionellen Outcome bei Patienten mit akutem Schlaganfall verbessert. Die Ergebnisse waren widersprüchlich. Deswegen haben die Kollegen aus Holland zu dieser Fragestellung die große randomisierte Studie PASS (Preventive Antibiotics in Stroke Study) initiiert.

Patienten und Methodik: Es handelt sich um eine multizentrische randomisierte offene Studie, bei der die Endpunkte verblindet evaluiert wurden. Eingeschlossen wurden Patienten mit akutem Schlaganfall, die ent-

Westendorp WF, Vermeij JD, Zock E et al; for the PASS investigators. The Preventive Antibiotics in Stroke Study (PASS): a pragmatic randomised openlabel masked endpoint clinical trial. Lancet 2015; DOI: http:// dx.doi.org/10.1016/S01406736(14)62456-9 weder über vier Tage mit täglich $2 \mathrm{~g}$ Ceftriaxon behandelt wurden oder ohne Antibiose verblieben. Der primäre Endpunkt war der funktionelle Outcome nach drei Monaten, gemessen mit der modifizierten Rankin-Skala. Sekundäre Endpunkte waren Tod, In- fektionsraten, Einsatz von Antibiotika und Dauer des Krankenhausaufenthaltes.

Ergebnisse: Zwischen Juli 2010 und März 2014 wurden insgesamt 2.550 Patienten in 30 Schlaganfallstationen in Holland aufgenommen. Jeweils 1.275 Patienten erhielten die Antibiose mit Ceftriaxon oder keine Antibiose.

Die Antibiose hatte keinen Einfluss auf den funktionellen Outcome nach drei Monaten ( $\vee$ Abb. 1). Die Odds Ratio (OR) für die modifizierte Rankin-Skala betrug 0,95. Nebenwirkungen waren in der aktiven Behandlungsgruppe kein größeres Problem. Für die Sterblichkeit, die jeweils $5 \%$ betrug, gab es keinen Unterschied. Auch für die Subgruppen im Alter unter und über 75 Jahren, für Untergruppen bezüglich ischämischen Insult, TIA oder zerebrale Blutung und Schwere des Schlaganfalls ergaben sich keine Unterschiede.

Schlussfolgerungen: Eine präventive Behandlung mit Ceftriaxon bei Patienten mit akutem Schlaganfall hat keinen Einfluss auf den funktionellen Outcome nach drei Monaten.

\section{- Kommentar von Hans-Christoph Diener, Essen}

\section{Antibiotikaprävention ist „passé"}

Mit der sehr großen und sehr gut durchgeführten randomisierten PASS-Studie dürfte die Diskussion um den Nutzen einer präventiven antibiotischen Behandlung bei Patienten mit akutem Schlaganfall erledigt sein. Damit sind auch die widersprüchlichen Ergebnisse der bisherigen kleinen Studie aus dem Weg geräumt.

Wichtig ist, dass die Ergebnisse sowohl bei Patienten mit ischämischem Insult als auch bei Patienten mit zerebralen Blutungen nachweisbar waren. Man kann also in Zukunft auf Stroke Units abwarten, bis eine Infektion eintritt und diese dann entsprechend antibiotisch behandeln.

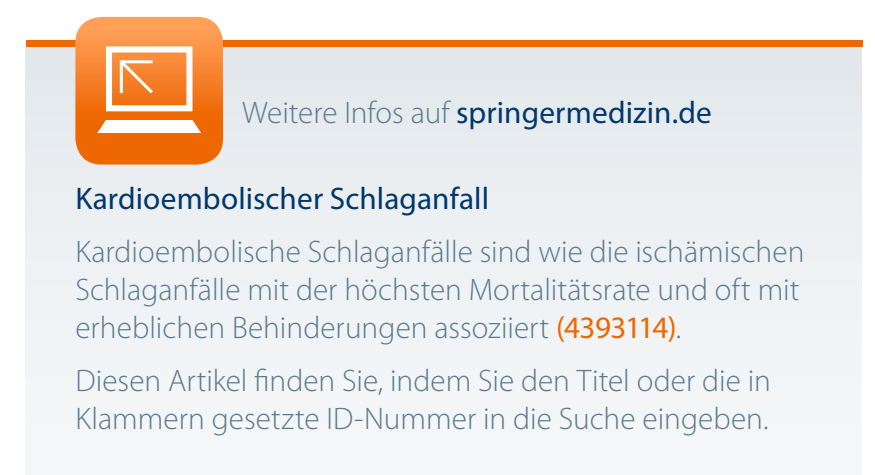

